\title{
Primary Ewing's Sarcoma of the Lumbosacral Spine: A Case Report
}

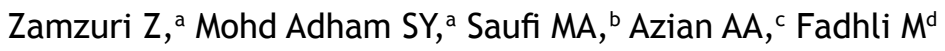 \\ ${ }^{a}$ Department of Orthopaedic, Traumatology \& Rehabilition, Kulliyyah of Medicine, International Islamic \\ University Malaysia, Kuantan, Pahang, Malaysia \\ ${ }^{b}$ Neurosurgical Division, Department of Surgery, Kulliyyah of Medicine, International Islamic University \\ Malaysia, Kuantan, Pahang, Malaysia \\ 'Department of Radiology, Kulliyyah of Medicine, International Islamic University Malaysia, Kuantan, \\ Pahang, Malaysia \\ ${ }^{d}$ Department of Pathology, Hospital Tengku Ampuan Afzan, Kuantan, Pahang, Malaysia
}

\begin{abstract}
Ewing's sarcoma is a rare tumor first discovered by James Ewing in 1921. It is more common in bone or skeletal component compared to soft tissue or extraosseous Ewing's sarcoma. Among soft tissue Ewing's, spinal cord involvement is rarer with only nine cases reported. We report a case of nine-year-old Malay girl who presented with low back pain for two months following a fall with progressive neurological deficits of bilateral lower limb. Magnetic resonance imaging was suggestive of a well-defined margin of intradural extramedullary tumor. With nerve sheath tumor in mind, surgical excision with laminectomy L2-S1 was performed. Intraoperative finding was an extradural mass from L3-L5 with extension to bilateral neuroforamen. Histopathology report defined a round cell tumour of Ewing's sarcoma from the mass.
\end{abstract}

KEYWORDS: Extradural, Ewing's sarcoma, primary spine tumor, cord compression

\section{INTRODUCTION}

Ewing's sarcoma is a rare, highly malignant tumour that primarily presents in the second decade of life with a slightly male predilection. It accounts for one-quarter of all primary bone tumors during childhood.Although thistumourusuallyaffectsthelong bones, it can also develop elsewhere. Ewing's sarcoma has been reported in a variety of extraosseous sites, including the larynx, scalp, nasal region, neck and lung. However, the incidence of primary spinal extradural Ewing's sarcoma is very low. To date, only nine cases have been reported in the English medical journal. Here, we describe a case of primary extradural Ewing's sarcoma with emphasis on clinical presentation, radiological features and management.

\section{CASE REPORT}

AH, a 9- year- old Malay girl complained of low back pain for two months following a fall during a sport

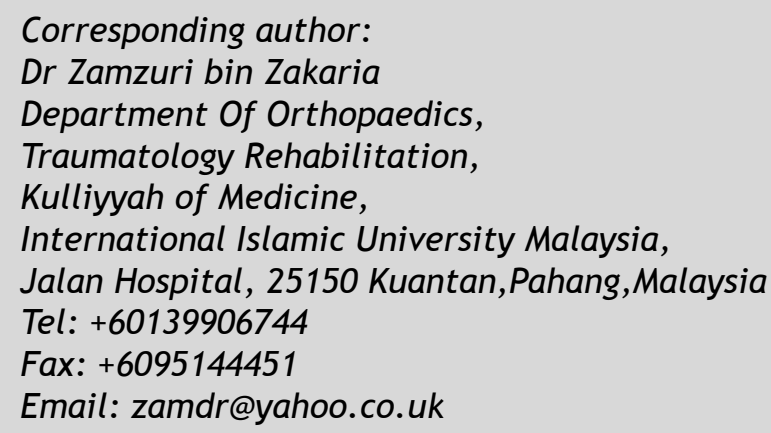

activity in school. The pain was pricking in nature, deep seated, localized, mild in severity but later progressively became worse. She was unable to sleep at night and had to lean forward while walking to get relief from the pain. Her condition was associated with weakness of her right lower limb. One week prior to admission, she experienced urinary incontinence, constipation and was unable to walk due to worsening of weakness of both the lower limbs. There was no history of fever, and no constitutional symptoms present. There was no history of Tuberculosis exposure. She completed compulsory immunization schedules, including the BCG.

Clinically, she was not cachexic or anaemic. There was a gibbus at the lumbar region with a kyphotic deformity of the spine. Both thighs showed wasting of the quadriceps muscle, which was worse over the right side. There was a flexion deformity of the right hip at $60 \mathrm{o}$. There was no bony or paraspinal muscle tenderness over the thoracolumbar area. Neurological examination of the lower limbs revealed reduced motor power of the knee flexion and extension to grade 4, ankle dorsiflexion and plantarflexion to grade 3 and the extensor and flexor hallucis longus. Sensory was intact bilaterally. Reflexes of the knee and ankle jerk were hyporeflexia. There was no clonus and the Babinski test was negative. Perianal sensation was intact, but the anal tone was lax.

Her hemoglobin level was $13.1 \mathrm{~g} / \mathrm{dl}$ with normal total white cell count. Erythrocyte sedimentation rate (ESR) was $80 \mathrm{~mm} /$ hour, and the Mantoux test was not 
reactive. Plain radiograph of the lumbosacral spine showed a kyphotic deformity between L4/L5 with no evidence of bony destruction. Magnetic Resonance Imaging (MRI) of the lumbosacral spine showed an intradural extramedullary mass extending and causing expansion of the neural foramen at L4-S1 levels suggestive of a nerve sheath tumor (Figure 1).

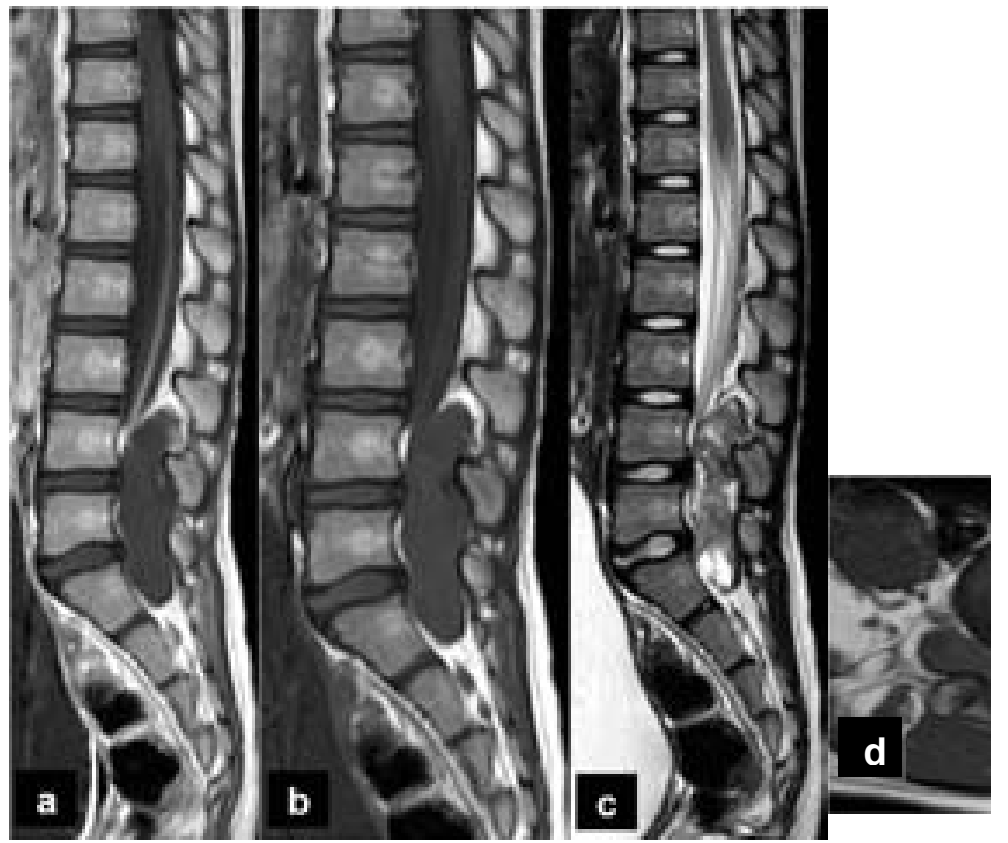

Figure 1: MRI in sagital views - (a) T1W, (b) T1 post IV Gadolinium, (c) T2W; showing the lobulated non-enhancing intradural extramedullary mass at L4 to S1 levels. The mass is hypointense on T1 and has mixed signal on T2. The spine is intact.(d) MRI axial T1W - the mass is shown to extend through and cause widening of the right neural foramen and to a lesser extent to the left side at L4 to S1 levels.

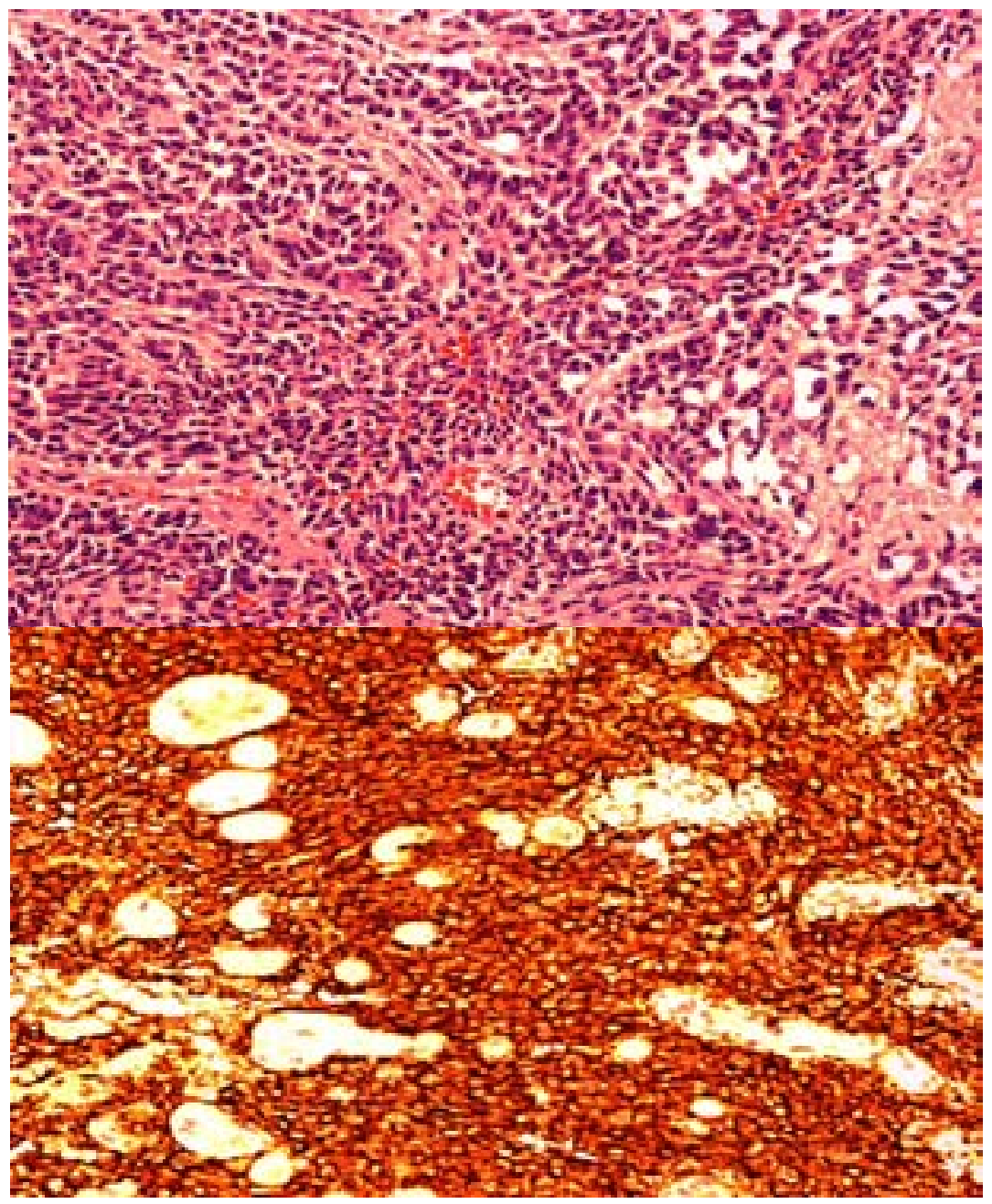

Figure 2: (a) Round and oval shape of tumor cells in H\&E staining. (b)Positive tumor cells in CD99 special staining. 


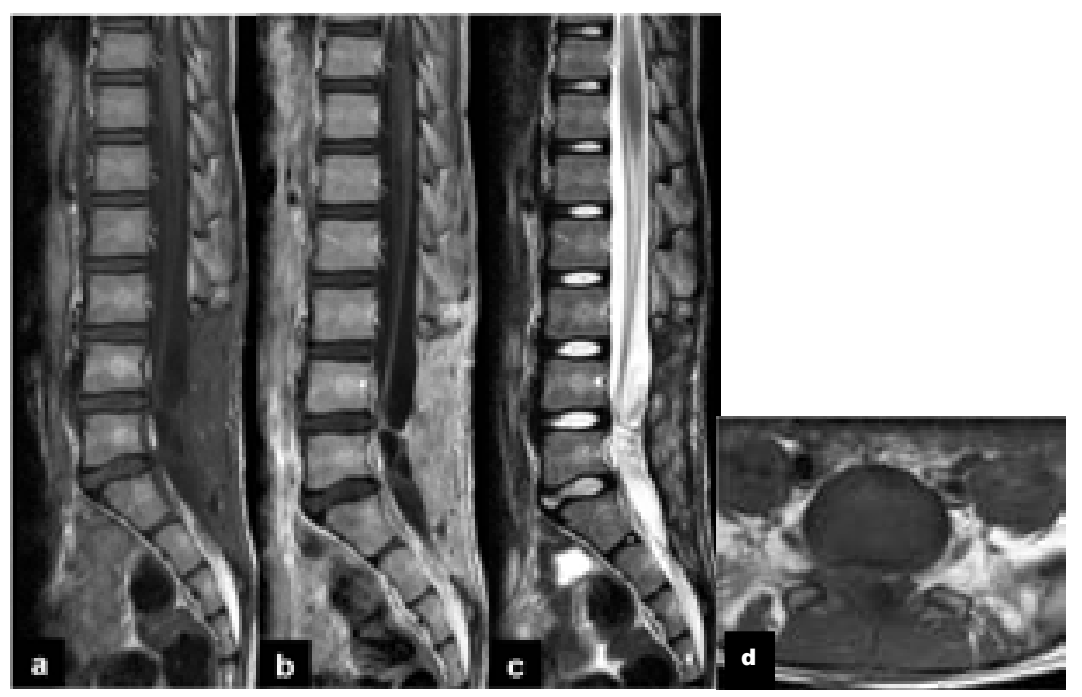

Figure 3: Post-operative MRI in sagital views - (a) T1W, (b) T1 post IV Gadolinium, (c) T2W. Slight expansion of the posterior thecal sac from L4 to S1 levels following the laminectomy at L4-L5.(d)Axial T1WHypointense lesion on T1 (heterogenous on T2 with minimal enhancement post IV Gadolinium - images not shown) is demonstrated within the L5 and S1 neural foramen, more on the right side.

Laminectomy from L2 until the S1vertebra with a radical tumor excision was done. Intraoperative findings revealed an extradural mass which was well encapsulated, greyish with cystic areas. The mass extended from L2 until the S1 vertebra with bilateral neuroforamen extension from L3 to L5 vertebra. Histopathological examination of the mass revealed small to medium in sized cells with hyperchromatic round to oval in shape. The immunohistochemistry CD 99 tests were positive with a non resistant periodic acid Schiff -diastase special staining tests (Figure 2). The features are indicative of Ewing's sarcomas. Post operative MRI of the lumbosacral spine showed slight expansion of the posterior thecal sac from L4 to S1 level following the laminectomy at the L4L5 with evidence of residual lesions noted within the right L5S1 neural foramen (Figure 3).

AH was then referred to a paediatric oncology centre and was enrolled for chemotherapy using EURO EWING TRIAL 99 protocol. She had undergone an "induction chemotherapy," combinations of vincristine, ifosfamide, doxorubicin and etoposide (VIDE) followed by one cycle of VAI (vincristine, actinomycin D and ifosfamide). She responded well to the chemotherapy, and subsequently; she will receive another 7 more cycles of VAl to complete the treatment. The whole treatment will take several months. Once completed, she will be followed up monthly for six months, every two months for six months, every three months for a year, every six months for three years and once every year thereafter. She will only undergo radiotherapy if there are recurrent symptoms at follow-up.

\section{DISCUSSION}

Spinal cord tumors are rare and fortunately affect only a minority of the population worldwide. These tumors are classified based on their anatomical locations in relation to the dura mater and spinal cord as an extradural, intradural, extramedullary or volume 11 Number 1 June 2012 intramedullary. Extradural Ewing's sarcoma is extremely rare and affects primarily pre-adolescents and adolescents. Our patient presented with low back pain following a fall in school, which was associated with progressive bilateral lower limbs weakness, including bowel and bladder incontinence. Clinical manifestations of Ewing's sarcoma arising primarily in the spinal epidural space include back pain with or without radicular pain, paresis of one or both legs, sensory disturbances, and bladder and bowel dysfunction ${ }^{1,2}$ Clinically, there is kyphotic deformity of the spine where 30 percent of the patient having spinal deformities during presentation. ${ }^{3}$ Pyogenic infection of the spine and tuberculosis are among the differential diagnoses which should be ruled out. Herniation of the intervertebral disc may be the next important differential diagnosis besides malignancy, especially in adolescents and adults. ${ }^{4} \mathrm{As}$ our patient presented with a neurological deficit of the lower limbs, the cause of cord compression must be evaluated radiologically.

Plain radiograph of the lumbosacral spine showed kyphotic deformity at the L4 and L5 vertebra with no evidence of osteolytic or osteosclerotic changes of the vertebral body. Magnetic resonance imaging (MRI) of the lumbosacral spine showed an intradural extramedullary mass extending and causing expansion of the neural foramen at L4-S1 levels. Magnetic resonance imaging, especially the contrast-enhanced spinal MRI, is most useful for early diagnosis of tumors around the spinal column. Although MRI is nonspecific for determining the histology of malignant tumors, it is useful for determining the extent of local involvement with the surrounding structures.

After the tumor was delineated on MRI, laminectomy with tumor resection was necessary to relief the symptoms. Laminectomy was preferred in this case instead of laminoplasty because the mass had 
extended to the neural foramen at L3-L5 bilaterally. More importantly, this method allowed for wider resection and nerve root's identification. Posterior element stability remained intact as the facet joints were preserved. Posterior instrumentation was not warranted.

Treatment for Ewing Sarcoma is multimodal involving radiation, chemotherapy, and surgery. Radiation can be given externally or internally (brachytherapy).

External radiation passes through tissues to the tumor site. High-energy ionizing rays destroy cells directly during division and indirectly by damaging nuclear DNA. Adjuvant chemotherapy has reduced distant metastasis, especially to the lungs, in patients with Ewing's sarcoma. Chemotherapeutic agents destroy cells during the cell cycle. Surgery is the oldest recorded form of curative treatment for cancer. Surgery is used in the Ewing's sarcoma patient for diagnosis, treatment (primary, adjuvant, salvage, and pallative), second-lookprocedures, andreconstruction. Primary surgery was the preferred treatment for many years where both the tumor and large areas around the tumor site are removed.

A 5-year survival rate of extraskeletal Ewing's sarcoma has been reported to be between $38 \%$ and $67 \%$. However, the clinical outcomes of extraskeletal Ewing's sarcoma around the spinal column are very poor, even with both chemotherapy and radiotherapy. The 5-year survival rate in extraskeletal Ewing's sarcoma of the spinal canal has been reported to be between $0 \%$ and $37.5 \%$. This poor prognosis may result from the high rate of partial resections of the tumors. Partially resected tumors are associated with a greater incidence of local recurrence and a higher mortality. ${ }^{5,6}$ Therefore, it is important for the surgeon to remove as much of the tumor. In view of the late relapses that have been reported in patients with extraskeletal Ewing's sarcoma, a longer follow-up and larger series are both required to improve our understanding of the natural history of this disease.

\section{CONCLUSION}

Extradural Ewing's sarcoma is an extremely rare tumour. It is difficult to diagnose and also difficult to treat. Few reported cases showed that it responded well to radiation and chemotherapy besides surgical excision. Multimodal therapy improves survival rates provided early diagnosis is made.

\section{REFERENCES}

1. Goktepe AS, Alaca R, Mohur H, Coskun U. Paraplegia: an unusual presentation of Ewing's sarcoma. Spinal Cord 2002;40:367-9.

2. Kaspers GJ, Kamphorst W, van de Graaff $M$, van Alphen HA, Veerman AJ. Primary spinal epidural extraosseous Ewing's sarcoma.
Cancer 1991;68:648-54.

3. DeSousa AL, Kalsbeck JE, Mealey J Jr, Campbell RL, Hockey A. Intraspinal tumours in children. A review of 81 cases. J Neurosurg 1979;51:437-45.

4. Dogan S, Leković GP, Theodore N, et al. Primary Thoracolumbar Ewing's sarcoma presenting as isolated epidural mass. Spine 2009;9:e9-14.

5. Harimaya K, Oda Y, Matsuda S, et al. Primitive neuroectodermal tumor and extraskeletal Ewing sarcoma arising primarily around the spinal column: report of four cases and a review of the literature. Spine 2003;28:e408-12.

6. Kim SW, Shin H. Primary Intradural Extraosseous Ewing's Sarcoma. J Korean Neurosurg Soc 2009;45:179-81. 